Check for updates

Cite this: RSC Adv., 2019, 9, 14109

Received 30th January 2019

Accepted 3rd April 2019

DOI: 10.1039/c9ra00817a

rsc.li/rsc-advances

\section{Facile silicone oil-coated hydrophobic surface for surface enhanced Raman spectroscopy of antibiotics}

\author{
Muhammad Usman, $\dagger^{\mathrm{a}}$ Xin Guo, $\dagger^{\mathrm{a}}$ Qiusheng Wu, ${ }^{\mathrm{a}}$ Jitesh Barman, (D) ${ }^{\mathrm{b}}$ Shaoqiang Su, ${ }^{\mathrm{c}}$ \\ Bingru Huang, ${ }^{a}$ Tang Biao, ${ }^{b}$ Zhang Zhang (D) ${ }^{c}$ and Qiuqiang Zhan (D) *a
}

\begin{abstract}
Surface-enhanced Raman scattering (SERS) technique has emerged as a potentially powerful tool for the detection of trace amounts of environmental contamination and pollutants such as various antibiotics and their active metabolites in the surface aquatic ecosystem (drinking water). In this study, we report the detection method for ciprofloxacin and norfloxacin analytes, two largely used antibiotics in the world, at a very low detection concentration based on the enrichment and efficient delivery of analytes after the evaporation of the solvent on slippery-SERS substrates. The slippery-SERS substrates were fabricated in a very efficient and cost effective way by simply spin-coating the silicone oil onto the widely used glass slides followed by annealing. The analyte particles with gold nanorods (GNRs) were efficiently delivered to the active site by evaporating the aqueous solvent on the slippery surface via the suppression of the coffee ring effect caused by the smooth contraction motion of the base contact radius of the droplet without any pinning. Thus, the detection limits of ciprofloxacin and norfloxacin analytes were reduced to $0.01 \mathrm{ppm}$, which is the lowest limit of detection achieved by any SERS technique. Finally, this study suggests that the fabricated silicone oil-coated slippery surface and GNRs based combinational approach for the SERS detection technique might be a powerful strategy for the reliable detection of the aqueous pollutant analytes even at very low concentrations.
\end{abstract}

\section{Introduction}

Trace amount detection, which is one of the most fundamental requirements of analytical science, can be accomplished efficiently by highly sensitive plasmonic nanosensors integrated with Raman spectroscopy using an enhanced electric field that is present at hot spots on the surface of plasmonic nanostructures. ${ }^{\mathbf{1 - 4}}$ This technique enables the ultrasensitive detection of analytes at even down to few molecules along with high specificity, which is beneficial to various promising researches and applications such as pre-cancer diagnosis and treatment, forensic analysis, security and food safety. ${ }^{5-7}$ However, single molecule detection is inherently challenging as molecular dispersion and diffusion causes a poor signal-to-noise ratio. In surface-enhanced Raman spectroscopy (SERS), plasmonic nanostructures are widely used for low-concentration sensing of

${ }^{a}$ Centre for Optical and Electromagnetic Research, Guangdong Provincial Key Laboratory of Optical Information Materials and Technology, South China Academy of Advanced Optoelectronics, South China Normal University, 510006 Guangzhou, China. E-mail: zhanqiuqiang@m.scnu.edu.cn

${ }^{b}$ Electronic Paper Display Institute, South China Academy of Advanced Optoelectronics, South China Normal University, 510006 Guangzhou, China

'Institute of Advanced Materials, South China Academy of Advanced Optoelectronics, South China Normal University, 510006 Guangzhou, China

$\dagger$ These authors contributed equally to the work. analytes due to their ability to diffuse into the small liquid volume containing an ultra-trace amount of molecules, resulting in the enhancement of typical Raman signals over several orders of magnitude. SERS is known as an efficient upgradation of the conventional Raman spectroscopy with its built-in functionalities, including simplicity, non-compactness, label-free sensing capability and specificity, while eliminating its drawbacks such as auto fluorescence background interruptions and weak signals in biological analyte detections. ${ }^{8-11}$ Metal nanostructures, especially nanorods with tunable plasmonic resonances, can be used for maximum signal generation and thus enhancement by SERS. ${ }^{12-16}$ On this line, the delivery of preconcentrated samples, i.e., analyte molecules, to a welldeveloped sensor with a high degree of sensitivity as well as specificity is one of the fundamental method that can be implemented. Sample molecules can be concentrated using centrifugal force (centrifugation), electric field (electrophoresis), antibody-protein interaction (protein immunoblot or Western blot), phase partitioning (chromatography), diffusion rate gradient (membrane-based dialysis) or physical and chemical properties of molecules (solid-phase extraction (SPE)). ${ }^{17-19}$ A large starting volume is a disadvantage for these analytical techniques. This drawback can be overcome by using microfluidic devices, although it is a complicated and timeconsuming process. Alternative to the former process, analyte 
enrichment by droplet evaporation has been investigated widely due to its cost effectiveness and extensive applicability to numerous functional nanomaterials. ${ }^{20}$ In most studies, superhydrophobic surfaces were used for droplet evaporation due to their dynamic ability of analyte delivery to SERS-active sites. ${ }^{21,22}$ However, the super hydrophobic surfaces for such applications suffer from the drawback of maintaining the stability of air pockets between the surface structures. The air pockets could easily be damaged by external wetting pressure and could lose wetting property by damaging the surface structure. ${ }^{23}$ The super hydrophobic surface might be wetting for liquids having low surface tension except a special geometry is employed to the roughness. $^{24,25}$ Recently, liquid-infused slippery surfaces have emerged as a better alternative to the superhydrophobic surfaces as a liquid repellent surface with very low contact angle hysteresis due to their atomically smooth and chemically homogeneous liquid lubricant surface. ${ }^{26}$ Very few studies in the existing literature reported on the evaporation-based analyte detection by SERS technique using the slippery surface. All the studies used superhydrophobic/hydrophobic surfaces, which were typically fabricated by the combination of surface structures and their functionalization with a low surface energy coating, as a substrate to infuse the lubricant for the fabrication of slippery surfaces. An extra step in the complicated fabrication of hydrophobic/superhydrophobic surface, which can be a very expensive and time-consuming process, is required for the SERS detection. To our knowledge, almost no study has been reported on the detection of analytes using the SERS technique based on the evaporation method on inexpensive hydrophilic substrates consisting of oxide surface groups such as glass.

In this study, we present a simple method for the fabrication of a highly sensitive SERS substrate based on the hydrophilic substrate. Herein, a lubricating liquid-infused slippery surface was prepared by spin coating the silicone oil on a clean glass slide, which was then used for reversing the coffee ring effect during the sessile droplet evaporation of an aqueous dispersion of GNRs (synthesized by the wet chemical method) and analyte molecules at a low concentration. Two representative fluoroquinolones, ciprofloxacin (CIP) and norfloxacin (NOR), which are widely used for both humans and animals, have been chosen as analytes to be detected at very low concentrations. Evaporation of droplets on this non-pinning platform results in the development of a densely packed array of GNRs with the analyte residing in their nanogaps. This strategy being efficient and cost-effective makes the analyte detection possible by selfdelivery to SERS-active sites. This novel strategy of fabricating the SERS substrate can not only eliminate the complexities of tedious nanofabrication but also simplify the SERS detection procedure.

\section{Materials and methods}

\subsection{Materials}

Chloroauric acid $\left(\mathrm{HAuCl}_{4} \cdot 4 \mathrm{H}_{2} \mathrm{O}, 99.99 \%\right)$ was bought from Sinopharm Chemical Reagent Co. China. Hexadecyltrimethyl ammonium bromide (CTAB, 97.0\%), silver nitrate $\left(\mathrm{AgNO}_{3}\right.$, $99.8 \%)$, sodium borohydride $\left(\mathrm{NaBH}_{4}, 96 \%\right)$, and ascorbic acid
(AA, 99.6\%) were purchased from Aladdin $®$. Standards of norfloxacin and ciprofloxacin were purchased from Sigma-Aldrich. All chemicals were used without further purification. We used de-ionized (DI) water in all experiments. Dimethyl silicone was purchased from KESHI, China.

\subsection{GNRs preparation and characterization}

The employed GNRs were synthesized using a seed-mediated growth method according to our previous protocol. ${ }^{12}$ The seed solution was synthesized first. At room temperature, $\mathrm{HAuCl}_{4}$ $(0.1 \mathrm{~mL} ; 0.025 \mathrm{M})$ and CTAB $(5 \mathrm{~mL} ; 0.2 \mathrm{M})$ were mixed in a $20 \mathrm{~mL}$ scintillation vial. The solution was subsequently vigorously stirred for $30 \mathrm{~s}$. Then, ice-cold $\mathrm{NaBH}_{4}(0.6 \mathrm{~mL} ; 0.01 \mathrm{M})$ was quickly injected into the solution. The solution changed color from yellow to brownish yellow. For the growth solution, CTAB ( $5 \mathrm{~mL}, 0.2 \mathrm{M})$ was added to $(0.05,0.10,0.15,0.20$, and $0.25 \mathrm{~mL})$ of $0.16 \mathrm{mM} \mathrm{AgNO}_{3}$ solution at $30^{\circ} \mathrm{C}$. To this solution, $0.2 \mathrm{~mL}$ of $0.025 \mathrm{M} \mathrm{HAuCl}_{4}$ was added. After gentle mixing, $70 \mu \mathrm{L}$ of $0.08 \mathrm{M}$ ascorbic acid was added. Ascorbic acid as a mild reducing agent changed the growth solution color from dark yellow to colorless. Finally, $12 \mu \mathrm{L}$ of the seed solution was added to the growth solution at $30{ }^{\circ} \mathrm{C}$. The GNRs were well formed after overnight growth at room temperature.

\subsection{Hydrophobic, superhydrophobic and silicone oil-based slippery surface preparation}

The glass slides were washed by sonication for 5 minutes in ethanol, acetone and toluene. For the preparation of the hydrophobic surface, the surface chemistry of the cleaned glass slide was modified by grafting a low surface energy monolayer of octadecyltrichlorosilane (OTS) following the standard method. ${ }^{27}$ For the preparation of superhydrophobic surfaces, we simply added $3 \mathrm{~g}$ of PDMS liquid into the crucible, and placed it in the muffle furnace at $350{ }^{\circ} \mathrm{C}$ for two hours. After cooling down to the room temperature, the glass was superhydrophobic. $^{28}$

For the preparation of a slippery surface, a drop of silicone oil (kinematic viscosity $\approx 370 \mathrm{cSt}$ ) was placed on a cleaned glass slide and spin coated at $1000 \mathrm{rpm}$ for 1 minute. This process was useful for the removal of excess lubricating oil. Then, the silicone oil-coated glass slide was annealed at a temperature of $150{ }^{\circ} \mathrm{C}$ for 90 minutes. This resulted in the modification of the surface chemical property of glass to hydrophobic as well as the formation of a uniform silicone oil coating on the glass slide.

\subsection{Analyte detection on slippery surfaces using SERS}

$1 \mathrm{~mL}$ solution of GNRs was centrifuged twice at $8000 \mathrm{rpm}$ for 10 minutes each. Norfloxacin and ciprofloxacin with a concentration of $100 \mathrm{ppm}$ were diluted to different concentrations. Then, a drop of $30 \mu \mathrm{L}$ of this solution was deposited on the substrate and evaporated at room temperature. For SERS measurement, all the experiments were performed with a $633 \mathrm{~nm}$ wavelength laser excitation. The laser power in our experiment was about $1.5 \mathrm{~mW}$. A Raman microscope (Renishaw inVia) with a CCD detector and an optical microscope using a $\times 50$ microscope 
objective with a numerical aperture (NA) value of 0.6 was used for detecting inelastically scattered radiation.

\section{Results and discussion}

A perfect platform for acquiring the desired analyte concentration by the evaporation technique should be hydrophobic with minimum contact angle hysteresis for the sample droplet. The lubricated liquid-infused slippery surface consisting of silicone oil-coated glass slide is a simple representation of this theme, as shown in Fig. 1. Spin coating followed by annealing of silicone oil on the glass slide, which has the hydrophilic property due to the high surface energy of silicon dioxide, modifies the surface energy of the glass via the covalent bonding of silicone oil molecules with the glass substrate. The mechanism of the covalent bonding of silicone oil molecules with the glass substrate due to annealing is schematically shown in Fig. 1a, which is also demonstrated by Pant et al. ${ }^{29}$ Hence, this would make the substrate hydrophobic with excellent slipperiness. The water droplets on the fabricated slippery surface sliding with a very small tilt angle suggests that a small gravitational force was enough to overcome the pinning force of the slippery surface. Upon the evaporation of the sample droplet with analyte particles on an atomically smooth lubricant-infused slippery surface, the particles get enriched to a very small spot, and hence make a perfect hotspot for SERS detection. The slippery platform plays a crucial role in the self-assembly and enrichment of GNRs. The sequential process of the fabrication of the slippery platform is shown in Fig. 1. The fabricated slippery platform possessed a low energy surface and excellent hydrophobicity, which helped to spontaneously arrange the GNRs in an orderly structure after the drying process to produce a superlattice array. During the evaporation of the droplet, the analyte molecules filled in the gaps of the GNRs superlattice array and hence formed a "hot spot" for Raman signal detection. The formation of the high-density "hot spots" tremendously enhanced the Raman signal. The immense enhancement significantly improves the limit of detection (LOD), holding a potential prospect for SERS assays.

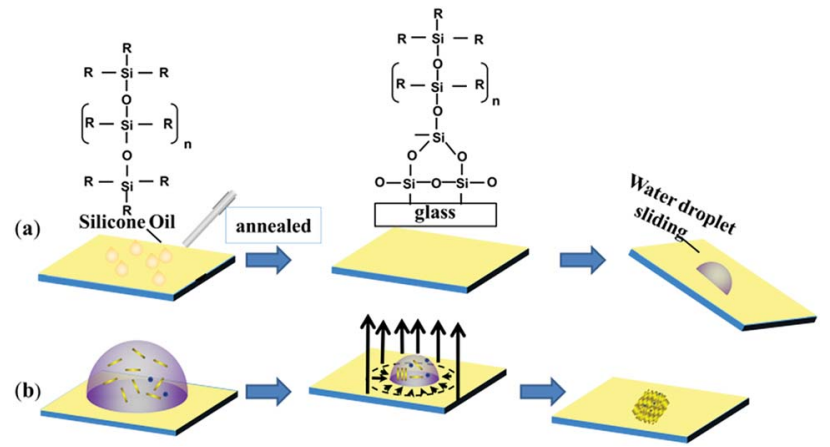

Fig. 1 Schematic of (a) the fabrication steps of slippery surface with the chemical structure of silicone oil and its bonding with the glass substrate and (b) the enrichment and concentration of Au nanorods and analyte molecules by an evaporation process.
The water repellency and smoothness of the slippery and nonslippery surfaces (hydrophilic, hydrophobic and superhydrophobic surfaces) were checked by contact angle $(\theta)$ and contact angle hysteresis $(\mathrm{CAH})\left(\Delta \theta=\theta_{\mathrm{a}}-\theta_{\mathrm{r}}\right)$, i.e., the difference between the advancing $\left(\theta_{\mathrm{a}}\right)$ and receding contact angle $\left(\theta_{\mathrm{r}}\right)$, which was measured by an optical goniometer. The high contact angle $\theta=110^{\circ}$ with a very low CAH $\Delta \theta=2^{\circ}$ on the slippery surface shows that the quality of the fabricated slippery surface is excellent. In terms of hydrophobicity, the nonslippery surfaces also exhibit good water repellency with a contact angle of $\theta=90^{\circ}$ for the hydrophobic surface and $\theta=150^{\circ}$ for the superhydrophobic surface; however, the hydrophilic surface shows very poor water repellency as the contact angle measured on the surface was $\theta<10^{\circ}$. The evaporation process of aqueous droplets $(30 \mu \mathrm{L})$ with GNRs and analyte particles was monitored on nonslippery and slippery glass substrates by tracking the drop contact diameter from the side using the goniometer (see Fig. 2). The droplet has a large initial contact diameter $(e)$ on nonslippery surfaces (hydrophilic) of $d=7 \mathrm{~mm}$ (corresponding area $A \approx 38.47 \mathrm{~mm}^{2}$ ) due to the hydrophilic nature of the nonslippery surface. The contact diameter does not change much over the evaporation time and results in the spreading of particles over a large spot with a diameter of $5.6 \mathrm{~mm}$ (corresponding area $\approx 24.62 \mathrm{~mm}^{2}$ ) after complete drying of the solvent, as shown in Fig. 2a. However, the contact diameter of the droplet on the slippery surface is decreased significantly from its initial value of $4.454 \mathrm{~mm}$ (corresponding area $A \approx$ $15.59 \mathrm{~mm}^{2}$ ), which is lower compared to that of the same volume of the droplet on the non-slippery surface (hydrophilic) due to a very low CAH of the slippery surface, to $0.493 \mathrm{~mm}$ (corresponding area $\approx 0.19 \mathrm{~mm}^{2}$ ) after complete drying of the solvent, as shown in Fig. 2 b. Fig. $2 \mathrm{c}$ and $\mathrm{d}$ show that the droplet contact diameter of the hydrophobic surface also has a relatively

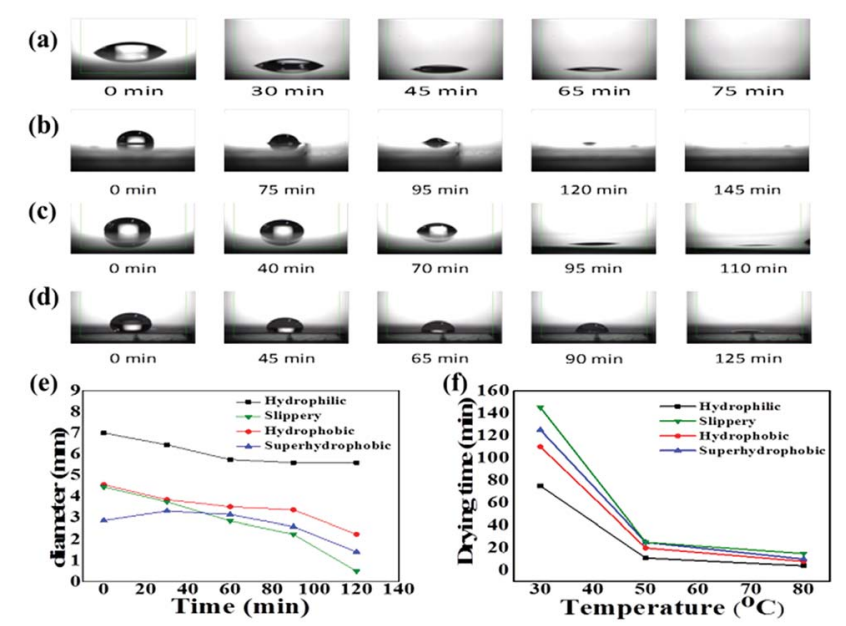

Fig. 2 Optical goniometer images showing droplet drying on (a) hydrophilic, (b) slippery, (c) hydrophobic and (d) superhydrophobic surfaces at different evaporation times. (e) The contact diameter (CD) of droplet on non-slippery (hydrophilic, hydrophobic and superhydrophobic) and slippery surface as a function of evaporation time. (f) Influence of temperature on droplet drying time for hydrophilic, hydrophobic, super hydrophobic and slippery surfaces. 
high initial value with $d=4.56 \mathrm{~mm}$ (corresponding area $\approx$ $16.32 \mathrm{~mm}^{2}$ ). After complete evaporation, the final contact diameter was $d=2.23 \mathrm{~mm}$ (corresponding area $\approx 3.90 \mathrm{~mm}^{2}$ ). However, in the case of the superhydrophobic surface, the initial diameter was small, i.e., $d=2.87 \mathrm{~mm}$, (corresponding area $\approx 6.47 \mathrm{~mm}^{2}$ ) due to its high contact angle. This is because the hydrophobic and slippery surfaces possess similar water repellency, whereas the superhydrophobic surface has a much higher contact angle. The final contact diameter of the superhydrophobic surface was $1.44 \mathrm{~mm}$ (corresponding area $\approx 1.63$ $\mathrm{mm}^{2}$ ). The contact diameter of all the surfaces varies with the evaporation time for all the surfaces, as shown in Fig. 2e. In the case of slippery surfaces, the reduction of contact area is very large at around $98.78 \%$, which is huge in comparison to those of the non-slippery surfaces (hydrophilic) $36 \%$ and $75 \%$ for the hydrophobic and super hydrophobic surfaces. The results show a similar trend with the highest decrease in contact diameter for slippery surfaces compared to those of the hydrophilic, hydrophobic and superhydrophobic surfaces due to the smaller pinning force provided by the slippery surface than the latter surfaces.

With the increase in temperature, the drying time decreases for all the substrates, confirming faster evaporation for all the substrates. However, the increasing temperature also enhances the surface pinning force for the evaporating drop on all the nonslippery surfaces, and the heat treatment negatively affects the analyte enrichment efficiency. For the slippery surface, only the drying time was influenced by the elevated temperature without any effect on the droplet enrichment efficiency, as shown in Fig. $2 \mathrm{f}$.

Fig. 3 shows the multiphoton laser scanning microscopy and SEM images, which suggest a similar interesting effect about the enrichment and the concentration of GNRs and analyte particles over the spot on both the surfaces after solvent drying. (a)
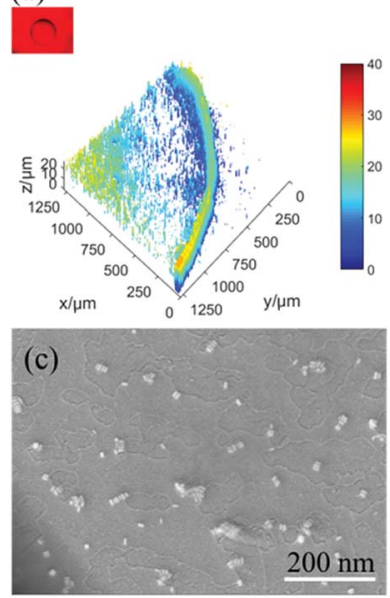

(b)
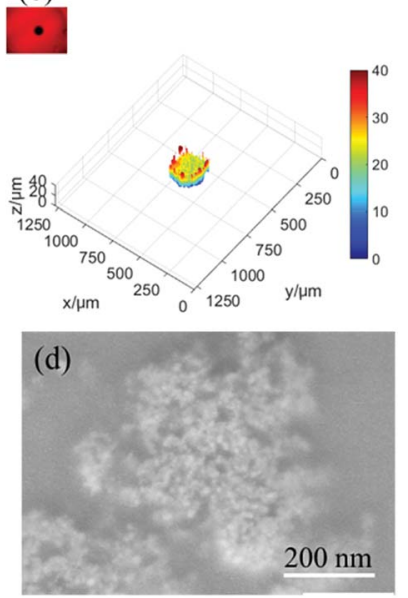

Fig. 3 Multiphoton laser scanning microscopic 3D images of evaporated GNRs and analyte solution on non-slippery (a) and slippery (b) substrates. The corresponding scanning electron microscopy (SEM) images of evaporated GNRs and analyte solution on non-slippery (hydrophilic) (c) and slippery (d) substrates.
The concentration of the particles on the non-slippery (hydrophilic) substrate follows a gradient pattern with the highest value at the edge of the droplet, which is known as the coffee ring effect. This effect is usually observed on the surfaces with hydrophilicity and pinning sites. However, the slippery surface exhibits a high concentration of particles over a very small spot by suppressing the coffee ring effect. The coffee ring effect, typically the accumulation of particles in the form of a ring after the evaporation of a droplet, arises from the pinning of the three phase contact line (TCL) at the droplet edge and causes an outward flow of the particles due to the gradient of evaporation rate from the middle to the edge of the droplet. ${ }^{30}$ The hydrophilicity and the heterogeneities (chemical or topographic) of the non-slippery surface caused the TCL pinning at the edge of the droplet during evaporation, which was confirmed by CA and $\mathrm{CAH}$ measurements. In the case of slippery surfaces, the TCL contracted very smoothly without any hindrance during the evaporation on the atomically smooth and chemically homogenous hydrophobic lubricant-infused surface. This easy contraction of the droplet without pinning on the slippery surface not only suppressed the coffee ring effect but also reversed it to enrich all the analyte particles to a very small spot. The SERS detection of the analytes was done at the hot spots after drying of solvents on both the surfaces. The morphology and structure of the particle assembly on non-slippery (hydrophilic) and slippery surfaces after the evaporation were characterized by scanning electron microscopy (SEM). For nonslippery surfaces, the detection spot was chosen at the edge of the coffee ring and can be seen clearly in Fig. 3a and c, which shows that the resulting GNRs and analyte particles after complete evaporation on a non-slippery surface depict a scattered pattern of the GNRs with a very small concentration of analyte molecules. As a result of this widespread pattern over a large area, the detection of analyte molecules became very difficult. We achieved remarkable results by the self-assembly of the GNRs and analyte solution on a slippery substrate. The pattern possesses a 3D disk-like morphology as shown in Fig. 3b. The SEM images in Fig. 3d reveals successful dense selfassembly of the GNRs into a homogeneous macroscopic 3D superlattice array, in which all the GNRs are dense as a result of particle enrichment via droplet evaporation on the lubricated liquid-infused slippery surface.

Fig. 4a shows the schematic of the molecular structure of norfloxacin, which is widely used as an antibiotic for diseases caused by Gram-positive ( $\mathrm{G}^{+}$) and Gram-negative ( $\mathrm{G}-$ ) bacteria as it inhibits the activity of DNA gyrase A in both humans and animals. ${ }^{31,32}$ Herein, Raman spectra measurements of highly diluted solutions of norfloxacin as the analyte molecule were conducted. Raman spectra measurement was carried out on the spot after the complete evaporation of the aqueous solvent of droplet $(30 \mu \mathrm{L})$ with GNRs and norfloxacin solutions. To find out the detection limit for norfloxacin, concentration of highly diluted sample solutions were systematically varied from 100 ppm, 10 ppm, 1 ppm, 0.1 ppm to $0.01 \mathrm{ppm}$.

Prominent Raman shifts of norfloxacin observed in Raman spectrograph are shown in Fig. 4b: (i) $485.6 \mathrm{~cm}^{-1}$ represents the bending vibration of $\mathrm{C}-\mathrm{C}$ of the aliphatic chain along with the 

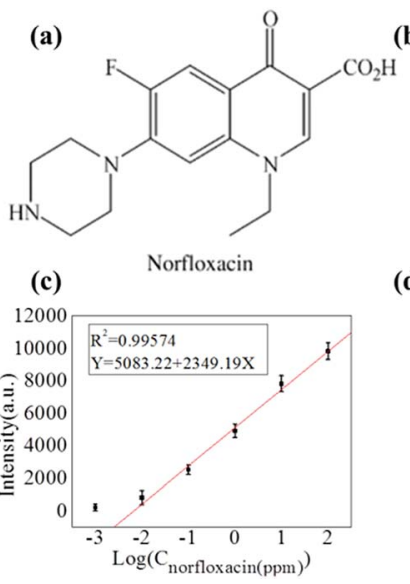

(d)
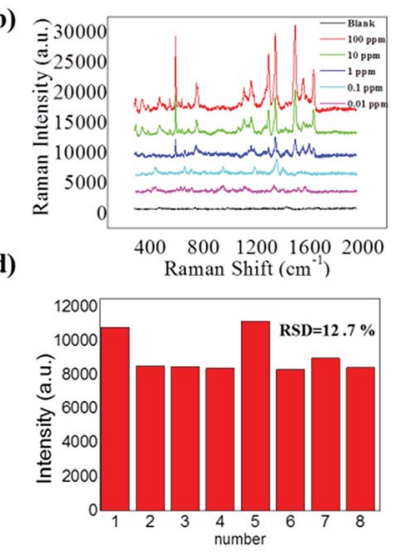

Fig. 4 (a) Schematic of the molecular structures of norfloxacin (NOR) (b) SERS spectra of norfloxacin on a slippery surface. (c) The corresponding intensity-concentration relationship extracted from the strongest peak of different concentrations. (d) Histogram of the peak SERS intensity at $1380 \mathrm{~cm}^{-1}$ of NOR acquired from 8 different spots 3D-superlattice arrays

C-N stretching vibration of the piperazinyl group; ${ }^{33}$ (ii) $760.7 \mathrm{~cm}^{-1}$ demonstrates the symmetric stretching vibration of the $\mathrm{C}-\mathrm{F}$ group; ${ }^{34}$ (iii) $1380 \mathrm{~cm}^{-1}$ shows the symmetric stretching vibration of the $\mathrm{O}-\mathrm{C}-\mathrm{O}$ group of carboxylic acid and methylene deformation mode of the piperazinyl group; and (iv) the peak at $1511.1 \mathrm{~cm}^{-1}$ is the symmetric stretching of the carbonyl group of the pyridone moiety and the stretching vibration of the (C-C) aromatic ring chain. Also, the peak at $1655.1 \mathrm{~cm}^{-1}$ indicates the $\mathrm{N}+\mathrm{H} 2$ scissoring of the piperazinyl group. ${ }^{33-35}$ The resulting Raman signals elevated gradually with the increased concentration of norfloxacin, as shown in Fig. $4 \mathrm{~b}$. The black line in the graph is of the solution in which no norfloxacin was added. It can be seen that no clear peaks for norfloxacin were found. These results depict that there exists a relatively excellent linear relationship between norfloxacin concentration and the signal intensity of SERS.

A calibration curve for the characteristic Raman peak of norfloxacin at $1380 \mathrm{~cm}^{-1}$ as a function of concentration was constructed to illustrate the sensitive performance of enrichment of analytes on the slippery surface. As shown in Fig. 4b, the Raman signal increased gradually with the increase in the norfloxacin concentration. Fig. $4 \mathrm{c}$ shows the calibration curve of the intensity-concentration relationship extracted from the characteristic peak of norfloxacin at various concentrations for nine repeated experiments for every concentration. The limit of detection of norfloxacin using our method was as low as $0.01 \mathrm{ppm}$. Importantly, it possessed a relatively excellent linear relationship (correlation coefficient, $R^{2}=0.9957$ ) between the ratio of SERS signal and the norfloxacin concentration. In Fig. 3d, the point-to-point difference of the SERS intensity for the $1380 \mathrm{~cm}^{-1}$ peak from 8 point was analyzed and the RSD is approximately $12.7 \%$. As a result, the proposed strategy can be applied in quantitative analysis.

Another synthetic antibiotic ciprofloxacin (CIP) (molecular structure is shown in Fig. 5a), which is widely used for both
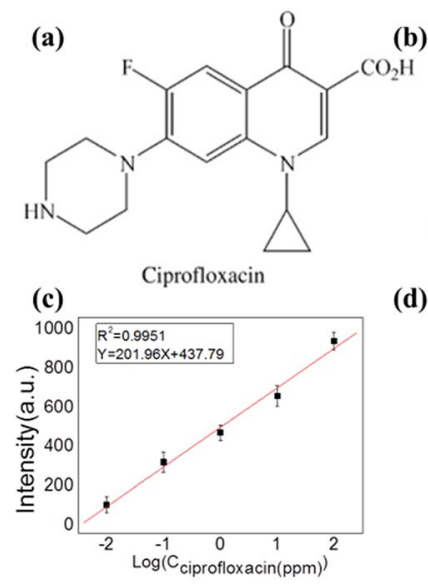

(d)
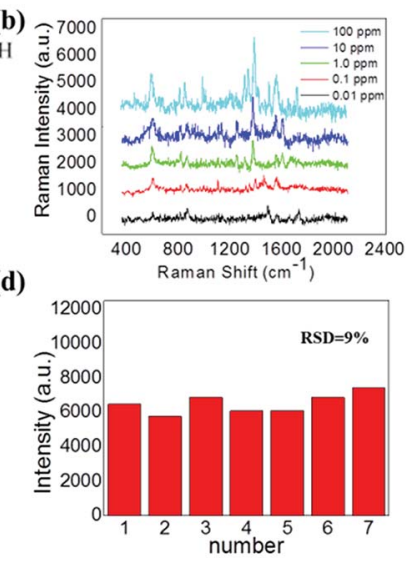

Fig. 5 (a) Schematic of the molecular structures of ciprofloxacin (CIPRO). (b) SERS spectra of ciprofloxacin on a slippery surface. (c) The corresponding intensity-concentration relationship extracted from the strongest peak of different concentrations. (d) Histogram of the peak SERS intensity at $1391 \mathrm{~cm}^{-1}$ of CIPRO acquired from 7 different spots 3D-superlattice arrays.

humans and animals was also used as an analyte in our experiment to demonstrate the multiformity of our method. A similar SERS study was carried out for different solution concentrations of CIP.

Prominent Raman shifts of ciprofloxacin observed in Raman spectrograph are shown in Fig. 5 b: the peak at $485.6 \mathrm{~cm}^{-1}$ is an evidence of the strong bending vibration of $\mathrm{C}-\mathrm{C}$ of the aliphatic chain and C-N stretching vibration, and the peak of $747 \mathrm{~cm}^{-1}$ indicates methylene rocking modes. The strong Raman band at about $1391 \mathrm{~cm}^{-1}$ is due to symmetric O-C-O stretching vibrations. The band located at $1581 \mathrm{~cm}^{-1}$ can be attributed to the symmetrical stretching vibration of the benzene ring.

During this process, the Raman peak of ciprofloxacin at $1391 \mathrm{~cm}^{-1}$ served as a characteristic peak for the construction of the calibration curve. In this procedure, the Raman signal increased gradually with the increasing concentration of ciprofloxacin, as shown in Fig. 5b. The corresponding calibration curves are shown in Fig. 5c. The limit of detection of ciprofloxacin using this strategy was as low as $0.01 \mathrm{ppm}$. Importantly, it also possessed a relatively excellent linear relationship (correlation coefficient, $R^{2}=0.9951$ ) between the ratio of SERS signal and the ciprofloxacin concentration. As a result, the proposed strategy can be applied multifariously in the quantitative analysis. The relative standard deviation (RSD) calculated by the variation of the SERS intensity for the $1391 \mathrm{~cm}^{-1}$ peak from the 7 different spots is found to be $9 \%$, as shown in Fig. 5 d.

However, $100 \mathrm{ppm}$ norfloxacin and ciprofloxacin was added into the GNRs solution and was evaporated on the non-slippery surface. SERS result for this pattern is shown in Fig. 6a and b, which implies that no significant Raman signals were obtained for norfloxacin and ciprofloxacin as compared to those obtained on lubricated liquid-infused slippery surface as a result of the non-enrichment of analyte particles on the non-slippery surface, (which was confirmed by SEM (Fig. 3c)). Norfloxacin 

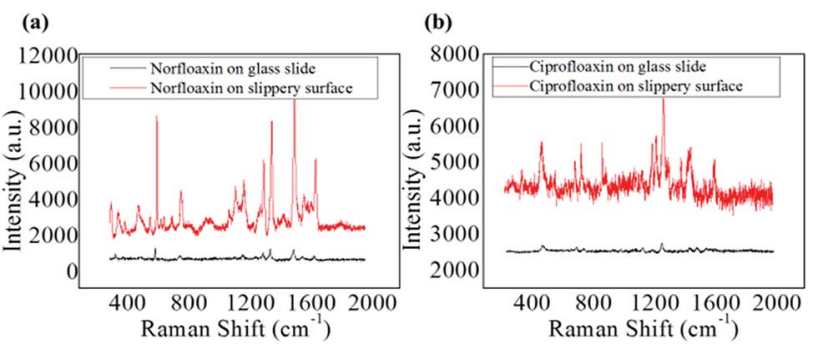

Fig. 6 (a) SERS spectra of norfloxacin on slippery surface and glass slide $100 \mathrm{ppm}$. (b) SERS spectra of ciprofloxacin on slippery surface and glass slide 100 ppm.

and ciprofloxacin with concentration lower than $100 \mathrm{ppm}$ showed no Raman signal on the non-slippery surface.

\section{Conclusion}

In summary, we demonstrated the single molecular analyte detection using the SERS technique based on the analyte and GNRs enrichment to make superlattices by solvent evaporation on slippery substrates. The substrates fabricated by a simple spin coating and annealing of silicone oil were cost effective hydrophilic substrates that contained the oxide surface group. The hydrophobicity and the absence of pinning sites on the slippery substrate not only suppressed the coffee ring effect but also provided a smooth surface for the three-phase contact line of the droplet to contract very easily. Thus, the process efficiently delivered the molecules inside the droplet to a concentrated 'hot spot', which was observed by optical microscopy and SEM. The dense aggregation of GNRs in a small area with analyte molecules inside the gaps tremendously enhanced the Raman signals, which enabled us to detect norfloxacin and ciprofloxacin down to a $0.01 \mathrm{ppm}$ concentration level. A calibration curve for the intensity of the characteristic Raman peak of the analyte that follows a linear relationship with the concentration with high repeatability is also presented. Therefore, we believe that our strategy for the trace detection of analytes could be used as an easy and simple alternative to expensive multi-step fabrication processes.

\section{Conflicts of interest}

There are no conflicts to declare.

\section{Acknowledgements}

This work was supported by the National Natural Science Foundation of China (61675071 and 61405062), the Guangdong Provincial Science Fund for Distinguished Young Scholars (2018B030306015), the Pearl River Nova Program of Guangzhou (201710010010), the Guangdong Innovative Research Team Program (201001D00104799318, 2011D039), the Natural Science Fund of Guangdong province (2014A030313445, 2018B030306015), Joint International Research Laboratory of Optical Information, the Key Laboratory of Optoelectronic
Devices and Systems of Ministry of Education and Guangdong Province (Shenzhen University) and the open fund of State Key Laboratory of Modern Optical Instrumentation of Zhejiang University (No. MOIKF201801).

\section{Notes and references}

1 B. Sharma, R. R. Frontiera, A.-I. Henry, E. Ringe and R. P. Van Duyne, Mater. Today, 2012, 15, 16-25.

2 J. Chen, M. Huang, Y. Zou, B. Song, Y. Wang, K. Wang, X. Li, X. Liu, X. Chen and F. Li, Laser Phys. Lett., 2016, 13, 105601.

3 X. Lin, Y. Cui, Y. Xu, B. Ren and Z. Tian, Anal. Bioanal. Chem., 2009, 394, 1729-1745.

4 K. A. Willets and R. P. V. Duyne, Annu. Rev. Phys. Chem., 2007, 58, 267-297.

5 S. Duraipandian, W. Zheng, J. Ng, J. J. Low, A. Ilancheran and Z. Huang, Anal. Chem., 2012, 84, 5913-5919.

6 S. Grilli, L. Miccio, O. Gennari, S. Coppola, V. Vespini, L. Battista, P. Orlando and P. Ferraro, Nat. Commun., 2014, 5, 5314.

7 J. Li, Y. Huang, Y. Ding, Z. Yang, S. Li, X. Zhou, F. Fan, W. Zhang, Z. Zhou and B. Ren, Nature, 2010, 464, 392.

8 P. E. Sheehan and L. J. Whitman, Nano Lett., 2005, 5, 803807.

9 P. Nair and M. Alam, Appl. Phys. Lett., 2006, 88, 233120.

10 F. Eftekhari, C. Escobedo, J. Ferreira, X. Duan, E. M. Girotto, A. G. Brolo, R. Gordon and D. Sinton, Anal. Chem., 2009, 81, 4308-4311.

11 J. Lin, J. Lin, Z. Huang, P. Lu, J. Wang, X. Wang and R. Chen, J. Innovative Opt. Health Sci., 2014, 7, 1350051.

12 Q. Zhan, J. Qian, X. Li and S. He, Nanotechnology, 2009, 21, 055704.

13 J. P. Juste, I. P. Santos, L. M. L. Marzán and P. Mulvaney, Coord. Chem. Rev., 2005, 249, 1870-1901.

14 B. Altansukh, J. Yao and D. Wang, J. Nanosci. Nanotechnol., 2009, 9, 1300-1303.

15 B. Sadeghi, F. S. Garmaroudi, M. Hashemi, H. R. Nezhad, A. Nasrollahi, S. Ardalan and S. Ardalan, Adv. Powder Technol., 2012, 23(1), 22-26.

16 W. Shi, J. Casas, M. Venkataramasubramani and L. Tang, ISRN Nanomater., 2012, 659043.

17 G. M. Walker and D. J. Beebe, Lab Chip, 2002, 2, 57-61.

18 R. A. Halvorson and P. J. Vikesland, Environ. Sci. Technol., 2010, 44(20), 7749-7755.

19 C. Zhao, Z. Ge and C. Yang, Micromachines, 2017, 8, 28.

20 P. Li, Y. Li, Z. Zhou, S. Tang, X. Yu, S. Xiao, Z. Wu, Q. Xiao, Y. Zhao and H. Wang, Adv. Mater., 2016, 28, 2511-2517.

21 F. D. Angelis, F. Gentile, F. Mecarini, G. Das, M. Moretti, P. Candeloro, M. Coluccio, G. Cojoc, A. Accardo and C. Liberale, Nat. Photonics, 2011, 5, 682.

22 F. Gentile, M. L. Coluccio, R. P. Zaccaria, M. Francardi, G. Cojoc, G. Perozziello, R. Raimondo, P. Candeloro and E. Di Fabrizio, Nanoscale, 2014, 6, 8208-8225.

23 M. Reyssat, J. Yeomans and D. Quéré, Europhys. Lett., 2007, 81, 26006.

24 T. Deng, K. K. Varanasi, M. Hsu, N. Bhate, C. Keimel, J. Stein and M. Blohm, Appl. Phys. Lett., 2009, 94, 133109. 
25 D. Quéré, Annu. Rev. Mater. Res., 2008, 38, 71-99.

26 L. Bocquet and E. Lauga, Nat. Mater., 2011, 10, 334.

27 J. Barman, A. K. Nagarajan and K. Khare, RSC Adv., 2015, 5, 105524-105530.

28 M. Long, S. Peng, X. Yang, W. Deng, N. Wen, K. Miao, G. Chen, X. Miao and W. Deng, ACS Appl. Mater. Interfaces, 2017, 9, 15857-15867.

29 R. Pant, P. K. Roy, A. K. Nagarajan and K. Khare, RSC Adv., 2016, 6, 15002-15007.

30 B. Agrawal, P. Chandra, R. N. Goyal and Y.-B. Shim, Biosens. Bioelectron., 2013, 47, 307-312.
31 J. Guan, G. Wells, B. Xu, G. McHale, D. Wood, J. Martin and S. S. Cole, Langmuir, 2015, 31, 11781-11789.

32 H. D. Silva, J. Pacheco, J. Silva, S. Viswanathan and C. D. Matos, Sens. Actuators, B, 2015, 219, 301-307.

33 Q. Tu, J. Eisen and C. Chang, J. Biomed. Opt., 2010, 15(2), 020512.

34 A. Gruodis, V. Aleksa, D. Powell, P. Klaeboe, C. Nielsen, G. Guirgis and J. Durig, J. Raman Spectrosc., 2003, 34, 711724.

35 A. Bright, T. R. Devi and S. Gunasekaran, Int. J. ChemTech Res., 2010, 2, 379-388. 